\title{
CLINICAL CHARACTERISTICS ASSOCIATED WITH DYSPHAGIA IN THE HOSPITALIZED ELDERLY
}

\author{
Y. Kuroda
}

\begin{abstract}
The aim of this study was to determine the factors associated with dysphagia in the hospitalized older adults. The dysphagia group consisted of 46 patients ( 23 men and 23 women) while the non-dysphagia group consisted of 40 patients (10 men and 30 women). The measurements included Mini Nutritional Assessment Short-Form (MNA-SF) scores, serum albumin levels, anthropometrics, and a mobility index. The dysphagia group was older and had significantly higher rates of male sex, respiratory disease on admission, dementia, other neurological disease, and impaired mobility than the non-dysphagia group. The dysphagia group also showed significantly lower values in nutritional measurements including MNA-SF scores, serum albumin levels. Logistic regression analysis showed that the factors significantly and independently associated with dysphagia were impaired mobility, dementia, and male gender. The results of present study showed that hospitalized elderly with dysphagia are likely to present with problems including physical and mental disabilities and malnutrition.
\end{abstract}

Key words: Dysphagia, elderly, hospitalization, disability, malnutrition.

\section{Introduction}

Dysphagia is a common condition among the elderly. Recent studies have reported the prevalence of oropharyngeal dysphagia as $27.2 \%$ in independently living elderly (1) and $47.4 \%$ in hospitalized elderly (2). Studies have also reported that dysphagia in the older population is associated with malnutrition (2-4), impaired activities of daily living $(1,4)$, increased rates of lower respiratory tract infection $(3,5)$, and higher mortality rates (5). These findings clearly indicate that dysphagia is a major health problem in this population.

One reason for the high prevalence of dysphagia in the elderly may be that diseases that can cause dysphagia increase with age (6). However, it is not uncommon to observe elderly patients developing dysphagia in the absence of any disease that is known to directly cause dysphagia. Another reason may be that a wide variety of stressors, such as acute illness, can induce dysphagia because the elderly have a diminished functional reserve available for swallowing (6). Although the latter hypothesis seems plausible, it does not fully explain the underlying mechanism considering that some patients have persistent or progressive dysphagia in the absence of acute illness. Thus, currently there are no well-

Department of Rehabilitation, St. Francis Hospital, Nagasaki City, Japan

Corresponding Author: Yoshitoshi Kuroda, Department of Rehabilitation, St. Francis Hospital, 9-20 Komine-machi, Nagasaki City, 852-8125 Japan, Telephone: +81-95846-1888, Fax: +81-95-845-7600, Email: kuroda@athena.ocn.ne.jp grounded hypotheses that can account for the underlying mechanism that result in the increased incidence of dysphagia in the elderly.

In order to provide effective intervention, further understanding of the underlying mechanism is mandatory. In the present study, we conducted a retrospective investigation comparing clinical characteristics between those with and without dysphagia among the hospitalized elderly. The aim of this study was to determine the factors associated with dysphagia in hospitalized older adults.

\section{Subjects and methods}

Subjects were retrospectively selected from patients admitted to a hospital for acute care between April 2013 and September 2014. Included were patients aged 65 years or older who were rated as having "malnutrition" or being "at risk of malnutrition" by the Mini Nutritional Assessment Short-Form (MNA-SF) (7) and who underwent a series of nutritional and physical assessments as described below. Excluded were those with active malignancy and those with feeding tubes. A total of 86 patients ( 33 men and 53 women) with a mean age (standard deviation [SD]) of 85.7 (7.7) years were enrolled as subjects. Primary diseases on admission were respiratory disease $(n=46)$, orthopedic disease $(n=8)$, digestive disease $(n=7)$, cardiac disease $(n=6)$, renal 
Table 1

Comparison between the dysphasia group and non-dysphagia group

\begin{tabular}{lccc}
\hline Variables & Dysphagia group $(\mathbf{n}=46)$ & Non-dysphagia group $(\mathbf{n}=40)$ & $\mathbf{p}$ value \\
\hline Gender (male) & $23(50.0 \%)$ & $10(25.0 \%)$ & 0.014 \\
Age (years) & $89.1 \pm 5.0$ & $81.7 \pm 8.3$ & $<0.001$ \\
85 or older & $38(82.6 \%)$ & $16(40.0 \%)$ & $<0.001$ \\
Respiratory disease (on admission) & $34(73.9 \%)$ & $12(30.0 \%)$ & $<0.001$ \\
Dementia & $32(69.6 \%)$ & $7(17.5 \%)$ & $<0.001$ \\
Other neurological disease & $21(45.7 \%)$ & $10(25.0 \%)$ & 0.047 \\
Calf circumference $(\mathrm{cm})$ & $23.5 \pm 3.8$ & $25.8 \pm 3.6$ & 0.006 \\
male $<31.3$; female $<29.9(\mathrm{~cm})$ & $44(95.7 \%)$ & $34(85.0 \%)$ & 0.090 \\
Mid-upper arm circumference $(\mathrm{cm})$ & $19.1 \pm 3.2$ & $20.1 \pm 3.2$ & 0.141 \\
male $<22.5 ;$ female $<20.8(\mathrm{~cm})$ & $35(76.1 \%)$ & $24(60.0 \%)$ & 0.109 \\
Mid-arm muscle circumference $(\mathrm{cm})$ & $17.4 \pm 2.4$ & $18.1 \pm 2.7$ & 0.206 \\
male $<19.1$; female $<16.6(\mathrm{~cm})$ & $29(63.0 \%)$ & $15(37.5 \%)$ & 0.018 \\
Serum albumin levels $(\mathrm{g} / \mathrm{dl})$ & $2.7 \pm 0.5$ & $3.0 \pm 0.6$ & 0.003 \\
$<2.8$ (g/dl) & $24(52.2 \%)$ & $11(27.5 \%)$ & 0.016 \\
Mini Nutritional Assessment score & $3.2 \pm 2.7$ & $6.2 \pm 2.8$ & $<0.001$ \\
$<8$ (classified as malnutrition) & $40(87.0 \%)$ & $25(62.5 \%)$ & 0.008 \\
Mobility index $(0-6)$ & $1.4 \pm 1.8$ & $3.9 \pm 2.1$ & $<0.001$ \\
$<2$ (almost bedridden) & $30(65.2 \%)$ & $7(17.5 \%)$ & $<0.001$ \\
\hline
\end{tabular}

disease $(n=4)$, inactive cancer $(n=4)$, and others ( $n=$ 11). Patients who were referred to speech therapists for swallowing management during hospitalization were defined as the dysphagia group and those who had no problems with swallowing, as the non-dysphagia group. The dysphagia group consisted of 46 patients (23 men and 23 women) while the non-dysphagia group consisted of 40 patients (10 men and 30 women). The severity of dysphagia was evaluated using the food intake level scale, with a range from 1 (no oral intake and no swallowing training) to 10 (normal oral food intake) (8). The scores of the dysphagia group ranged from 3 to 9 (median $=5$ ) while all subjects in the non-dysphagia group scored 10.

Nutritional assessment included MNA-SF scores, calf circumference $(C C)$, mid-upper arm circumference (MUAC), mid-arm muscle circumference (MAMC), and serum albumin levels. Physical ability was evaluated with a mobility index, measured as follows: 0 , completely bedridden; 1 , able to sit with a little assistance; 2 , able to sit without assistance; 3 , able to move from bed to wheel chair with a little assistance; 4 , able to move from bed to wheelchair without assistance; 5 , able to walk with a little assistance; and 6, able to walk without assistance. The two groups were compared for the age, sex, presence of respiratory disease (as a primary disease on admission), dementia, and other neurological diseases, and the above variables. In order to make the analyses more readily comparable, the continuous variables were converted into dichotomous variables based on cut off points. The cut-off points were applied for age ( 85 or older), MNA-SF score ( $<8$, suggesting malnutrition), CC $(<$ $31.3 \mathrm{~cm}$ for men and $<29.9 \mathrm{~cm}$ for women), MUAC $(<$ $22.5 \mathrm{~cm}$ for men and $<20.8 \mathrm{~cm}$ for women), MAMC $(<$ $19.1 \mathrm{~cm}$ for men and $<16.6 \mathrm{~cm}$ for women), and serum albumin levels $(<2.8 \mathrm{~g} / \mathrm{dL})$. The cut-off points of the anthropometrics were values 2 standard deviations below the normal population aged 40-44 years for each sex (9).

Comparative analyses between the two groups were performed using the Student's t-test, Mann-Whitney test, and chi square test. Logistic regression analysis was performed using dysphagia as the dependent variable and other dichotomous measures as independent variables. In the multivariate analysis, respiratory disease was excluded from the analysis because it is clearly a frequent result of dysphagia (5). Statistical significance was set at $\mathrm{p}<0.05$.

\section{Results}

Comparison between the dysphagia group and the non-dysphagia group is shown in Table 1 . The dysphagia group was older $(p<0.001)$ and had significantly higher rates of male sex $(p=0.014)$, respiratory disease on admission $(p<0.001)$, dementia $(p<0.001)$, other neurological disease $(p=0.047)$, and impaired mobility 
Table 2

Variables associated with dysphagia (multivariate logistic regression analysis)

\begin{tabular}{lccc}
\hline & Odds ratio & $\mathbf{9 5 \%}$ confidence interval & p value \\
\hline Mobility index $(<2)$ & 13.70 & $3.43-54.80$ & $<0.001$ \\
Dementia & 11.00 & $3.01-40.10$ & $<0.001$ \\
Sex (male) & 6.81 & $1.69-27.50$ & 0.007 \\
MAMC (below the cut-off point) & 3.14 & $0.92-10.80$ & 0.068 \\
\hline
\end{tabular}

$(\mathrm{p}<0.001)$ than the non-dysphagia group. The dysphagia group also showed significantly lower MNA-SF scores $(\mathrm{p}<0.001)$ and serum albumin levels $(\mathrm{p}=0.03)$, but no significant differences in MUAC $(\mathrm{p}=0.141)$ and MAMC $(p=0.206)$ measures. The results from the analysis using dichotomous variables were similar to those from the above analysis using continuous variables with the exception that the dysphagia group exhibited a significantly higher rate of decreased MAMC compared with the non-dysphagia group $(p=0.018)$, and the difference in the rate of decreased CC did not reach a significant level $(p=0.090)$.

Logistic regression analysis showed that the factors significantly and independently associated with dysphagia were impaired mobility (OR 13.70 95\% CI 3.4354.80), dementia (OR $11.095 \%$ CI 3.01-40.10), and male gender (OR 6.81 95\% CI 1.69-27.50) (Table 2).

\section{Discussion}

The dysphagia group was older than the nondysphagia group, which was consistent with previous studies $(1-3,5)$. It is of note that our results showed that male gender was significantly associated with dysphagia, which was inconsistent with the findings from Spanish studies $(1-3,5)$. Considering that a Korean study (10) and a Japanese study (11) also reported the association of male gender with dysphagia, such an association may be related to ethnicity.

Dementia and neurological disease were significantly associated with dysphagia which was consistent with previous findings $(2,5)$. However, such an association was not observed in a study in independently living elderly (3), which was probably related to the very low prevalence $(<10 \%)$ of each condition in the study cohort. Therefore, we consider that the association between dysphagia and dementia or neurological condition is typically apparent only in clinical populations.

The dysphagia group exhibited significantly impaired mobility compared to the non-dysphagia group. Furthermore, the multivariate analyses showed that impaired mobility was the most important predictor of dysphagia. Given that many studies have also demonstrated the association between dysphagia and activities of daily living in the elderly (1-5), the relationship between swallowing impairment and physical disability is of importance. Recent studies have proposed that sarcopenia is a common underlying cause of impaired physical ability and dysphagia (12-14). Further investigations regarding the effects of the loss of muscle mass and strength on swallowing function are needed.

The dysphagia group showed significantly lower values in the nutritional measures of MNA-SF score and serum albumin levels, suggesting an association between dysphagia and malnutrition. Anthropometric assessment using dichotomous variables also showed that the dysphagia group had significantly higher rate of decreased MAMC, suggesting a reduction of muscle mass may be a predictor for dysphagia. The difference in CC lost significance after applying the dichotomous variables, which was probably related to the very high prevalence (85\% or more) of the decreased condition.

Taken together, the results of the present study showed that older patients with dysphagia are likely to present with impaired mobility, dementia, and malnutrition. The results were mostly consistent with recent findings (2-5). We consider that dysphagia in elderly patients is rarely a condition specific to the swallowing mechanism, and in many cases, it can be understood as part of a systemic decline including physical, mental, and nutritional deterioration. Currently, there is no sound evidence that traditional dysphagia intervention techniques are effective for this clinical population. Given that many countries are facing challenges associated with aging populations, there is a great need to clarify the underlying mechanism of dysphagia in the elderly in order to improve its prevention and treatment.

The limitations of this study were as follows: First, the study sample was small and obtained from one institution, which may limit the generalization of the results. Second, dysphagia was assessed only with an observational scale. Third, the cross-sectional design did not allow for causative analysis.

In conclusion, hospitalized elderly with dysphagia are likely to present with problems including physical and mental disabilities and malnutrition.

Conflicts of interests: The author has no conflict of interest to disclosure, and do not receive any funding for this study. 


\section{References}

1. Serra-Prat M, Hinojosa G, Palomera E, Arreola V, Clavé P. Prevalence of oropharyngeal dysphagia and impaired safety and efficacy of swallowing in independently living older persons. JAGS 2011; 59: 186-187.

2. Carrión S, Cabré M, Monteis R, Roca M, Palomera E et al. Oropharyngeal dysphagia is a prevalent risk factor for malnutrition in a cohort of older patients admitted with an acute disease to a general hospital. Clin Nutr 2015; 34: 436-442.

3. Serra-Prat M, Palomera M, Gomez C, Sar-Shalom D, Saiz A et al. Orpharyngeal dysphagia is a risk factor for malnutrition and lower respiratory tract infection in independently living older persons: a populationbased prospective study. Age Aging 2012; 41: 376-381.

4. Wakabayashi H, Matsushima M. Dysphagia assessed by the 10 -item eating assessment tool is associated with nutritional status and activities of daily living in elderly individuals requiring long-term care. J Nutr Health Aging 2015. doi:10.1007/s12603-015-0481-4.

5. Cabré M, Serra-Prat M, Force LI, Almirall J, Palomera E et al. Oropharyngeal dysphagia is a risk factor for readmission for pneumonia in the very old persons: observational prospective study. J Gerontol A Biol Sci Med Sci 2014; 69: 330-337.

6. Ney D, Weiss J, Kind A, Robbins J. Senescent swallowing: impact, strategy and interventions. Nutr Clin Pract 2009; 24: 395-413.

7. Kaiser ML, Bauer JM, Ramsch C, Uter W, Guigoz Y et al. Validation of the
Mini Nutritional Assessment short-form (MNA-SF): a practical tool for identification of nutritional status. J Nutr Health Aging 2009; 13: 782-788.

8. Kunieda K, Ohno T, Fujishima I, Hojo K, Morita T. Reliability and validity of a tool to measure the severity of dysphagia: the food intake level scale. J Pain Symptpm Manage 2913; 46: 201-206.

9. Hosoya N, Okada T, Muto Y, Yamamoru H, Tashiro T et al. Japanese anthropometric reference data 2001 (JARD 2001). Jpn J Nutr Assess 2002; 19(Suppl): 1-81 (Japanese).

10. Yang EJ, Kim MH. Lim JY, Paik NJ. Oropharyngeal dysphagia in a community-based elderly cohort: the Korean longitudinal study on Health and Aging. J Korean Med Sci 2013; 28: 1534-1539.

11. Furuta M, Komiya N. Akifusa S, Shimazaki Y, Adachi M et al. Interrelationship of oral health status, swallowing function, nutritional status and cognitive ability with activities of daily living in Japanese elderly people receiving home care services due to physical disabilities. Community Dent Oral Epidemiol 2013; 41: 173-181.

12. Kuroda Y. Relationship between swallowing function, functional and nutritional status in hospitalized elderly individuals. Int J Speech Lang Pathol Audiol 2014; 2: 20-26.

13. Wakabayashi H. Presbyphagia and sarcopenic dysphagia: association between age, sarcopenia, and deglutition disorders. J Frail Aging 2014; 3: 97-103.

14. Maeda K, Akagi J. Sarcopenia is an independent risk factor of dysphagia in hospitalized older people. Geriatric Gerontol Int 2015. doi:10.1111/ggi.12486. 